\title{
A Planar Metamaterial With Dual-Band Double-Negative Response at EHF
}

\author{
T. Funda Gündoğdu, Kaan Güven, Mutlu Gökkavas, Costas M. Soukoulis, and Ekmel Özbay
}

\begin{abstract}
We report the fabrication and electromagnetic characterization of a planar composite metamaterial (CMM) that is designed to achieve dual-frequency double-negative response at the lower end of the extremely high-frequency (EHF) band. The CMM is based on cut wire pairs and continuous wire elements. Dual-frequency operation is obtained by employing cut wire pairs of two different lengths within the unit cell of the CMM. The magnetic response of the cut wire pairs and the left-handed transmission band of the CMM are demonstrated by experiment and numerical simulations. It is found that the combined electric response of the dual-band CMM is complicated and imposes certain restrictions to the structure design in achieving true left-handed response at both designated frequencies.
\end{abstract}

Index Terms-Electromagnetic propagation, metamaterial, negative refractive index.

\section{INTRODUCTION}

$\mathbf{M}$ ETAMATERIALS are artificial structures that respond to the incident electromagnetic wave with tailored effective permittivity and permeability functions. A particular class of metamaterials has electromagnetically resonant elements that provide negative permittivity and permeability response, and thus comprise an effective medium with negative refractive index [1]. Frequently used element designs are periodic wires for negative permittivity $(\varepsilon<0)$, and split-ring resonators and cut wire pairs for negative permeability $(\mu<0)$ response [2], [3]. Due to these two distinct components, these designs are also called composite metamaterials (CMMs). The majority of the metamaterial studies focused on achieving the desired electromagnetic response at a single frequency band. Dual- or multifrequency response is reported at microwave using S-shaped resonators [4], and omega-shaped resonators [5],

Manuscript received June 9, 2009; revised July 17, 2009. First published October 2, 2009; current version published April 7, 2010. This work was supported by the European Union under projects EU-METAMORPHOSE, EU-PHOREMOST, EU-PHOME, and EU-ECONAM, and TUBITAK under the Project 105E066, Project 105A005, Project 106E198, and Project 106A017. The work of K. Güven was supported by GEBIP program of Turkish Academy of Sciences.

T. F. Gündoğdu is with the Found. for Research and Tech. Hellas, GR-700 13 Heraklion, Greece, and also with Nanotechnology Research Center, Bilkent University Ankara, Turkey (e-mail: tamara@ iesl.forth.gr).

K. Güven is with the Physics Department, Koc University, 34450 Istanbul, Turkey (e-mail: kguven@ku.edu.tr).

M. Gokkavas is with the Nanotechnology Research Center, Bilkent University, 06800 Ankara, Turkey (e-mail: mgokkavas@bilkent.edu.tr).

C. M. Soukoulis is with the Iowa State University, Ames, IA 50011 USA (e-mail: soukoulis@ameslab.gov).

E. Özbay is with the Physics Department, Nanotechnology Research Center, Bilkent University, 06800 Ankara, Turkey (e-mail: ozbay@bilkent.edu.tr).

Color versions of one or more of the figures in this paper are available online at http://ieeexplore.ieee.org.

Digital Object Identifier 10.1109/JSTQE.2009.2031618 at near-infrared [6], [7] and terahertz regimes [8], [9]. The multiband operation may be derived from a single-band CMM structure by superposition principle, but the combined electric and magnetic response in a multiband CMM design can differ significantly from that of the individual responses of its constituent elements. Therefore, investigating the electromagnetic response of the dual structure in detail can elucidate design considerations in order to improve their utilization in multiband applications.

In this paper, we report the design, fabrication, and experimental/simulated characterization of a dual-band CMM aimed to operate at the lower part of the extremely high-frequency (EHF) band. The CMM exhibits double negative (i.e., simultaneously $\varepsilon<0$ and $\mu<0$ ) at both its designated frequencies. Section II describes the design and fabrication of the CMM. The simulated and measured transmission spectra of the metamaterial are presented in Section III. The measured phase advance through the CMM is given in Section IV.

\section{Dual-Band Cut Wire Pair and Wire Metamaterial}

The metamaterial consists of dielectric layers that are patterned with an array of metal cut wire and continuous wire elements on either side. The dielectric layers are then stacked periodically to form a 1-D metamaterial [10], [11]. For propagation normal to the layer plane, the electric field vector is parallel to the cut wires and the magnetic field vector is perpendicular to the plane between the cut wire pairs (see Fig. 1). In this propagation direction, the electric field induces a plasma resonance on both cut wire pairs and continuous wires. Consequently, the dielectric response of the medium below the plasma cutoff frequency becomes negative below the plasma frequency

$$
\varepsilon(\omega)=1-\frac{\omega_{p}^{2}}{\omega^{2}} \quad \omega_{p}^{2}=\frac{2 \pi c^{2}}{d^{2} \ln \left(2 d / w_{l}\right)}
$$

where $d$ is the separation between the wires, $w$ is the width of wires, and $c$ is the speed of light in the dielectric/air medium. This plasma frequency changes significantly in the presence of cut wires in the CMM.

The magnetic field induces an inductive-capacitive resonance on the cut wire pairs. The resonance frequency $\omega_{m}$ depends on the geometrical parameters (in particular, the length) of the cut wire pairs and the dielectric constant of the separating layer. For a detailed formulation of this dependence, see [3] and [10]. The overall magnetic response can be represented through an effective permeability

$$
\mu_{\mathrm{eff}}=1-\frac{F \omega_{m}^{2}}{\omega^{2}-\omega_{m}^{2}+i \omega \Gamma} .
$$




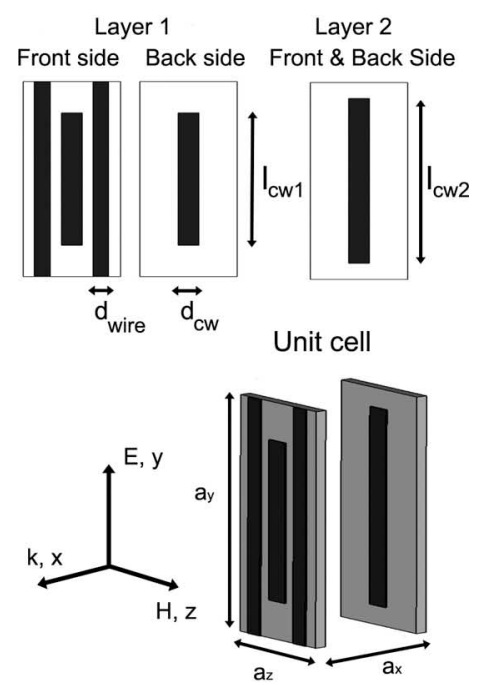

(a)

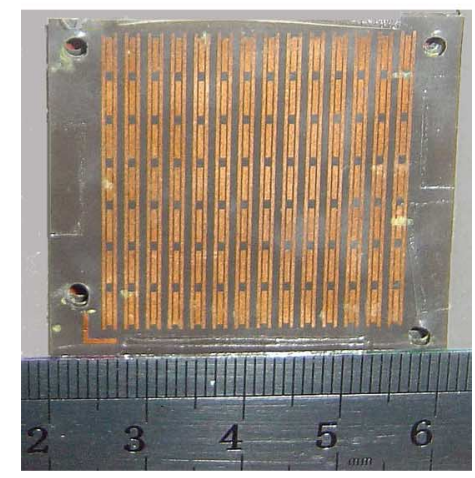

(b)

Fig. 1. (a) Schematic view of the dual-band metamaterial unit cell. (b) Sample photograph of the first layer showing array of cut wires and wires. The scale numbers are in centimeters.

TABLE I

Metamaterial Design Parameters

\begin{tabular}{|c|c|c|}
\hline Parameter & Value (mm) & Description \\
\hline$\overline{a_{x}}$ & 0.46 & unit cell period in $x$ \\
\hline$a_{y}$ & 4.50 & unit cell period in $y$ \\
\hline$a_{z}$ & 1.25 & unit cell period in $\mathrm{z}$ \\
\hline$l_{c w 1}$ & 2.90 & $1^{\text {st }}$ cutwire-pair length in $y$ \\
\hline$l_{c w 2}$ & 3.60 & $2^{\text {nd }}$ cutwire-pair length in $y$ \\
\hline$d_{c w}$ & 0.26 & cutwire-pair width in $z$ \\
\hline$d_{\text {wire }}$ & 0.20 & continuous wire width in $z$ \\
\hline$t_{\text {layer }}$ & 0.125 & dielectric layer thickness in $x$ \\
\hline
\end{tabular}

For dual-band operation, the unit cell is designed to accommodate two cut wire pairs of different length. The unit cell consists of two layers, each containing one cut wire pair. The continuous wires are located in one of the layers. Fig. 1 shows the schematic of the unit cell and a photograph of first layer. The geometrical parameters are given in Table I.

The cut wire and wire patterns are fabricated by chemical etching the thin copper layer on both sides of the dielectric layer. In addition, several layers are fabricated that do not include continuous wires so that the isolated response of the cut wire pairs can be investigated.

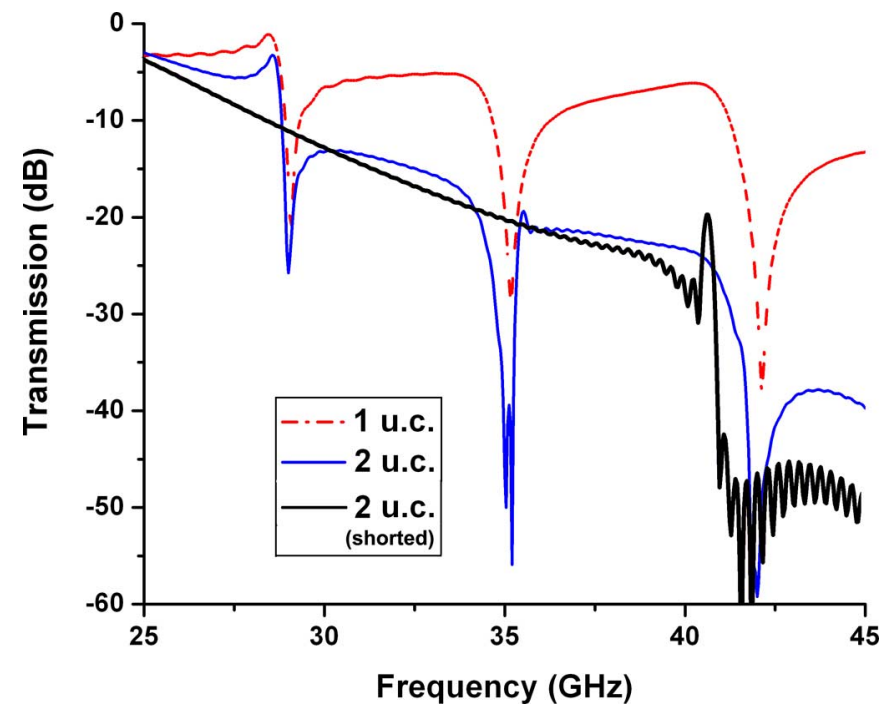

Fig. 2. Simulated transmission spectra of dual-band cutwire-pairs with one (top dashed line) and two (middle solid line) unit cells. The transmission of the electrically shorted cutwire-pair is given for comparison (bottom solid line).

It may be possible to design a truly modular structure where the operation frequencies of the metamaterial can be changed simply by inserting or extracting different cut wire pair layers. However, the nontrivial electric response of the CMM imposes certain restrictions on the design parameters in achieving double-negative response.

\section{Simulated Transmission OF THE DuAl-Mode CMM}

The simulated transmission spectra are obtained by using a commercial EM-solver software. The simulation employs electric and magnetic boundary conditions on the unit cell along the $y$ - and $z$-directions (see Fig. 1).

Fig. 2 shows the transmission spectrum of the dual cut wire pair medium containing one and two unit cells, respectively, in $x$-direction. The continuous wires are not present in this structure. The resonances occur at 28.2, 35.0, and 42.3 GHz. In order to exploit the origin of the resonance, the cut wire pairs are electrically shorted at their long ends. This removes the capacitive coupling at the ends, and hence, the magnetic coupling, but the electric coupling remains. The transmission spectrum of shorted cut wire pairs (black curve) clearly indicates that the first two resonances are of magnetic origin, whereas the resonance at 42.3 GHz is due to electric coupling. The suppression of the transmission thereafter in the two-unit-cell structure is associated with the photonic bandgap effects induced by the stacking periodicity [12].

The transmission spectrum of the dual-band CMM for one unit cell is shown in Fig. 3 (squares) with the cut wire pair spectrum (triangles). The CMM has three transmission peaks slightly shifted from the respective resonance dips of the cut wire pair. The shift occurs in the magnetic resonance frequency due to the presence of continuous wires. In order to identify the transmission peaks, a control specimen for the CMM is constructed by using cut wire pairs electrically shorted at their long ends. This shorted CMM exhibits only one transmission 


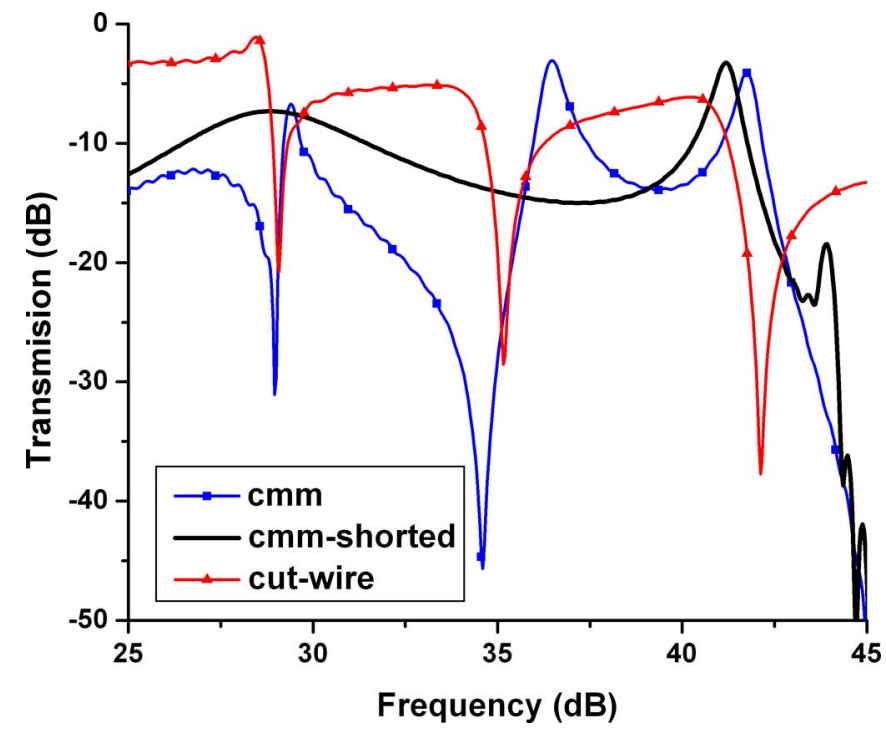

Fig. 3. Simulated transmission spectra of the CMM (squares) and the CMM with shorted cutwire-pairs (solid curve). The spectrum of cutwire-pair from Fig. 2 is included for comparison (triangles).

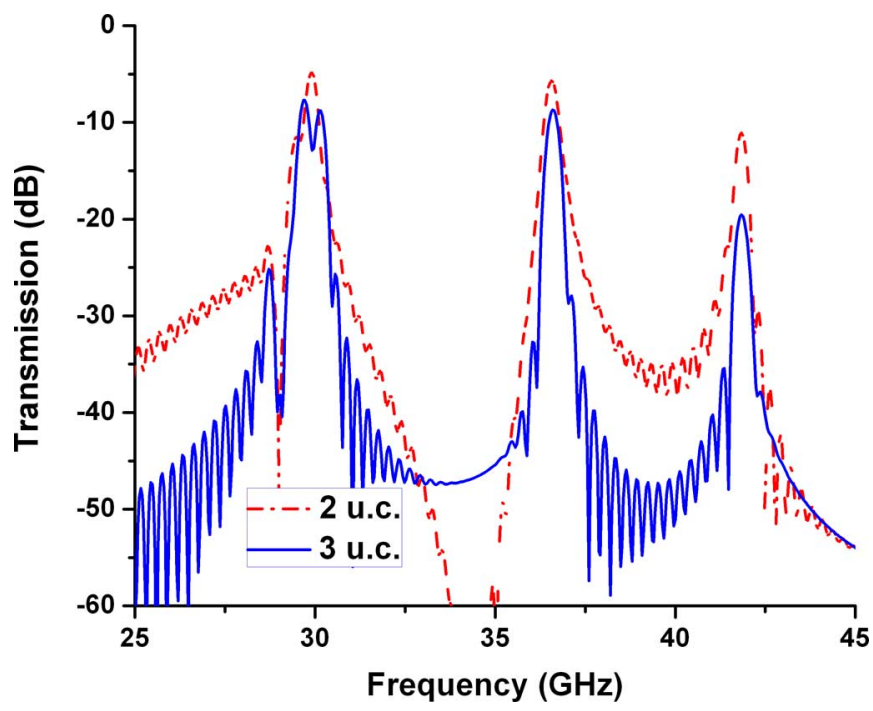

Fig. 4. Simulated transmission spectra of the CMM containing two (dashed line) and three (solid line) unit cells.

peak in common with that of the normal CMM [13]. Note that this peak is also common with the weak transmission peak of the cut wires at $\sim 41.0 \mathrm{GHz}$. By virtue of these results, the CMM comprises a double-negative $(\varepsilon<0, \mu<0)$ medium at its transmission bands around 28.2 and $35.0 \mathrm{GHz}$, and a positive $(\varepsilon>0, \mu>0)$ medium at $\sim 41 \mathrm{GHz}$.

Fig. 4 shows the transmission spectra of the CMM structures containing two and three unit cells, respectively. With increasing CMM thickness (i.e., number of layers), the transmission bands become relatively pronounced by the strong suppression of the background. The suppression originates from the loss inherent to the resonance (in particular, to the imaginary part of the dielectric permittivity).

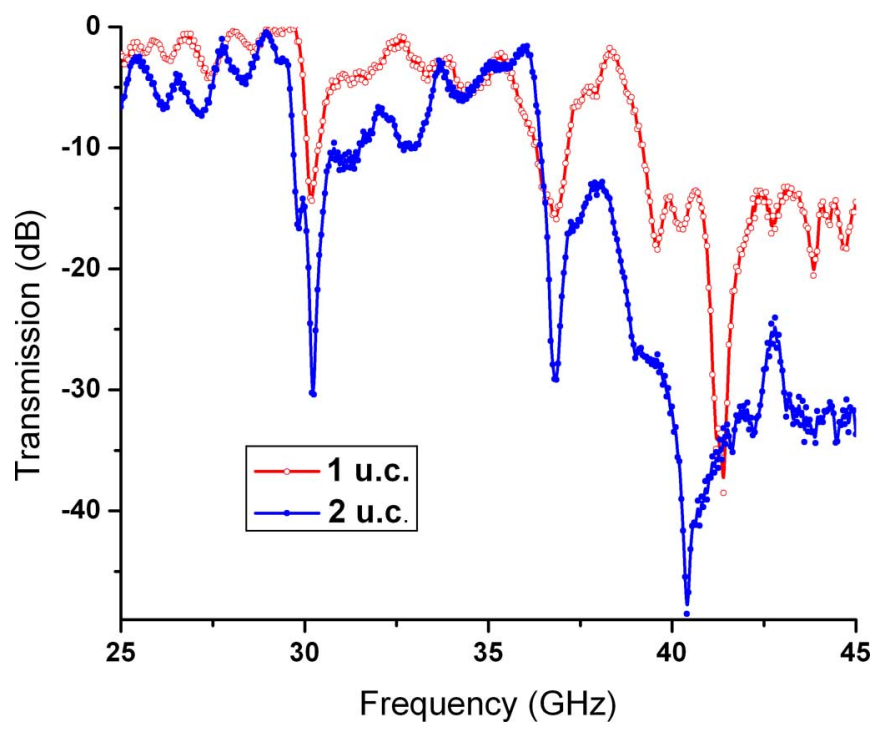

Fig 5. Measured transmission spectra of dual-band cut wire structure containing (top curve) one and (bottom curve) two unit cells.

\section{EXPERIMENTAL MEASUREMENTS OF THE DUAL-BAND CMM}

\section{A. Transmission Spectrum}

The measurement setup consists of an HP 8510C network analyzer and two horn antennas. Transmission measurements are performed in free space. In Fig. 5, the transmission of the dualband cut wire pair is shown. The resonances occur at 30,36, and $40.5 \mathrm{GHz}$. The electric resonance shows a slight shift for the two-unit-cell structure that is caused by the nonuniformity of the spacing of thin dielectric layers. The two-unit-cell structure exhibits the photonic bandgap effect next to the electric resonance in agreement with the simulations. The dependence of this gap on the spacing of layers will be discussed elsewhere [12].

Fig. 6 shows the measured transmission spectra of the dualband CMM with one and two unit cells. In agreement with the simulations (see Figs. 3 and 4), the transmission bands are obtained. The background suppression is also observed experimentally with increasing number of unit cells. Experiment data indicate that the misalignment between the layers affects the transmission level significantly.

\section{B. Phase Spectra}

We finally commence an analysis of the phase delay through the CMM with successive increasing number of unit cells. It is known that the phase advance is negative in a $(\varepsilon<0$ and $\mu<0)$ medium [14]. Thus, increasing the number of layers should decrease the phase dispersion at the transmission bands where the medium is double negative. Fig. 7 shows the phase dispersion for the CMM structures containing two, three, and four unit cells, respectively. The inset shows the behavior in the first $(30 \mathrm{GHz})$ transmission band, and the main figure shows the behavior at the second $(36 \mathrm{GHz})$ and third $(40.5 \mathrm{GHz})$ transmission bands. Evidently, there is a crossover between the second and third transmission bands where the ordering of the dispersion curves 


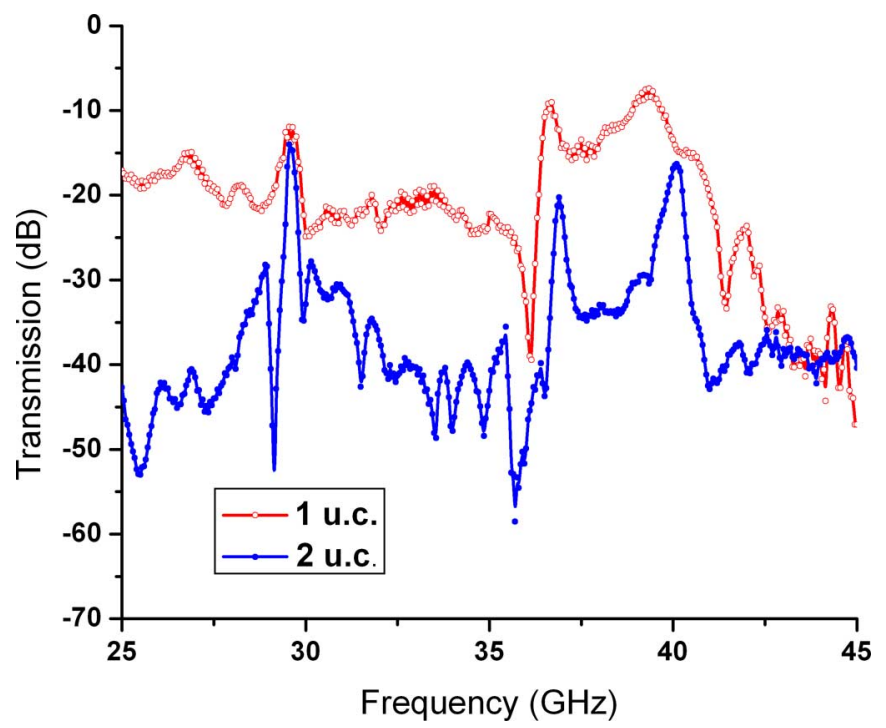

Fig 6. Measured transmission spectra of the CMM containing (top curve) one and (bottom curve) two unit cells.

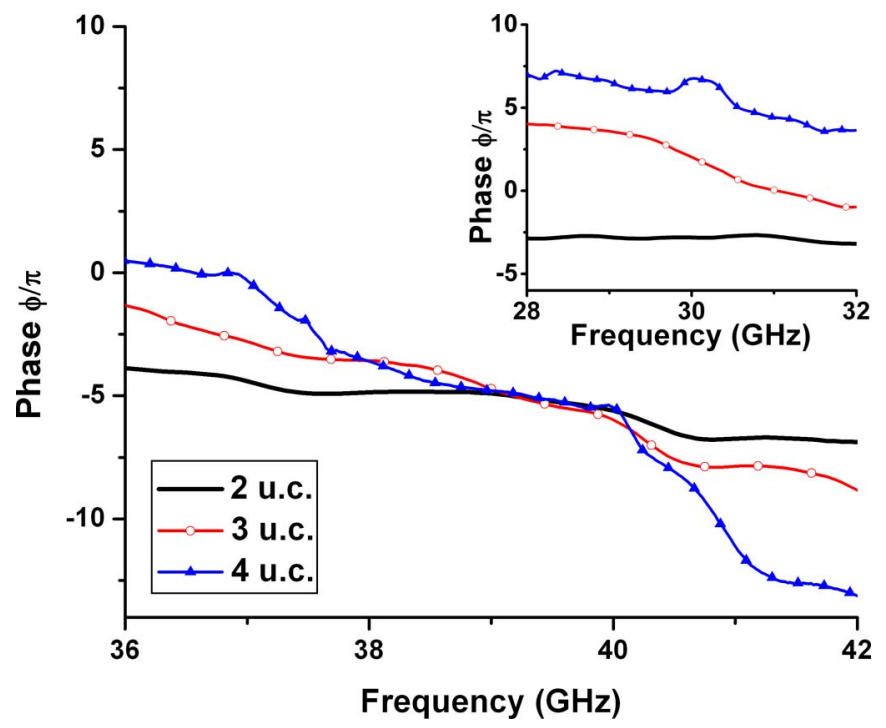

Fig. 7. Measured phase spectra of CMM structures containing two, three, and four layers of unit cell. Note the crossover of the phase dispersion curves before the last transmission band at $\sim 40 \mathrm{GHz}$.

is reversed. We observe that with increasing thickness of the CMM, the phase delay decreases (in magnitude) in the first and second bands, whereas it increases in the third band.

\section{CONCLUSION}

In this study, we present an experimental realization of a dualband CMM at EHF frequencies. We have found that although the dual operation can be based on a straightforward extension of single-band structures, the multilayer design of the CMM results in a nontrivial electric response, which must be taken into account to attain a proper double-negative $(\varepsilon<0$ and $\mu<0)$ medium response. Well-defined transmission bands are obtained with "thin" CMM structures containing few number of unit cells. When compared with in-plane propagating metamaterials [4], [5], the normal-to-plane propagation provides full beam coverage within a single layer. Some modularity is available in comparison to integrated "fishnet"-type structures reported in [7]-[9].

\section{ACKNOWLEDGMENT}

The authors thank K. B. Alici for fabrication of samples.

\section{REFERENCES}

[1] D. R. Smith, W. J. Padilla, D. C. Vier, S. C. Nemat-Nasser, and S. Schultz, "Composite medium with simultaneously negative permeability and permittivity," Phys. Rev. Lett., vol. 84, pp. 4184-4187, 2000.

[2] J. B. Pendry, A. J. Holden, W. J. Stewart, and I. Youngs, "Extremely low frequency plasmons in metallic mesostructures," Phys. Rev. Lett., vol. 76, pp. 4773-4776, 1996.

[3] J. B. Pendry, A. J. Holden, D. J. Robbins, and W. J. Stewart, "Magnetism from conductors and enhanced nonlinear phenomena," IEEE Trans. Microw. Theory Tech., vol. 47, no. 11, pp. 2075-2084, Nov. 1999.

[4] H. Chen, L. Ran, J. Huangfu, X. Zhang, K. Chen, T. M. Grzegorcyzk, and J. A. Kong, "Metamaterial exhibiting left-handed properties over multiple frequency bands," J. Appl. Phys., vol. 96, pp. 5338-5340, 2004.

[5] K. Aydin, Z. Li, M. Hudlicka, S. A. Tretyakov, and E. Ozbay, "Transmission characteristics of bianisotropic metamaterials based on omega shaped metallic inclusions," New J. Phys., vol. 9, pp. 326-336, 2007.

[6] D. Kwon, D. H. Werner, A. V. Kildishev, and V. M. Shalaev, "Near-infrared metamaterials with dual-band negative-index characteristics," Opt. Exp., vol. 15, pp. 1647-1652, 2007.

[7] U. K. Chettiar, A. V. Kildishev, H. Yuan, W. Cai, S. Xiao, V. P. Drachev, and V. M. Shalaev, "Dual-band negative index metamaterial: Double negative at $813 \mathrm{~nm}$ and single negative at $772 \mathrm{~nm}$," Opt. Lett., vol. 32, pp. 1671$1673,2007$.

[8] Y. Yuan, C. Bingham, T. Tyler, S. Palit, T. H. Hand, W. J. Padilla, D. R. Smith, N. M. Jokerst, and S. A. Cummer, "Dual-band planar electric metamaterial in the terahertz regime," Opt. Exp., vol. 16, pp. 9746-9752, 2008.

[9] M. Li, Z. Wen, J. Fu, X. Fang, Y. Dai, R. Liu, X. Han, and X. Qiu, "Composite metamaterials with dual-band magnetic resonances in the terahertz frequency regime," J. Phys. D, vol. 42, pp. 115420-1-115420-4, 2009.

[10] J. Zhou, L. Zhang, G. Tuttle, T. Koschny, and C. M. Soukoulis, "Negative index materials using simple short wire pairs," Phys. Rev. B, vol. 73, pp. 041101-1-041101-4, 2006.

[11] K. Guven, D. Caliskan, and E. Ozbay, "Experimental observation of left-handed transmission in a bilayer metamaterial under normal-to-plane propagation," Opt. Exp., vol. 14, pp. 8685-8693, 2006.

[12] T. F. Gundogdu, M. Gokkavas, K. Guven, Z. Li, and E. Ozbay, "Electric response in planar metamaterials," submitted for publication.

[13] K. Aydin, K. Guven, M. Kafesaki, L. Zhang, C. M. Soukoulis, and E. Ozbay, "Experimental observation of true left-handed transmission peak in metamaterials," Opt. Lett., vol. 26, pp. 2623-2625, 2004.

[14] K. Aydin, K. Guven, C. M. Soukoulis, and E. Ozbay, "Observation of negative refraction and negative phase velocity in left-handed metamaterials," Appl. Phys. Lett., vol. 86, pp. 124102-1-124102-3, 2005.

Authors' photographs and biographies not available at the time of publication. 